\title{
Collision-Induced Unfolding of Native-like Protein Ions Within a Trapped Ion Mobility Spectrometry Device
}

\author{
Nicholas B. Borotto*, Kemi E. Osho, Talitha Kamakamakamae Richards, and Katherine A. Graham \\ Department of Chemistry, University of Nevada, 1664 N. Virginia Street, Reno, Nevada 89557, United States \\ *E-mail: nborotto@unr.edu
}

\begin{abstract}
Native mass spectrometry and collision-induced unfolding (CIU) workflows continue to grow in utilization due to their ability to rapidly characterize protein conformation and stability. To perform these experiments, the instrument must be capable of collisionally activating ions prior to ion mobility spectrometry (IMS) analyses. Trapped ion mobility spectrometry (TIMS) is an ion mobility implementation that continues to grow in utilization due to its inherently high resolution and reduced instrumental footprint. In currently deployed instruments, however, typical modes of collisional activation do not precede IMS analysis and thus, the instruments are incapable of performing CIU workflows. In this work, we expand on a recently developed method of activating protein ions within the TIMS device and explore its analytical utility toward the unfolding of protein ions. We demonstrate the unfolding of native-like ions of ubiquitin, cytochrome $\mathrm{C}, \beta$-lactoglobulin, and carbonic anhydrase. These ions undergo extensive unfolding upon collisional activation. Additionally, the improved resolution provided by the TIMS separation uncovers previously obscured unfolding complexity.
\end{abstract}

Analyses of protein three-dimensional structure with mass spectrometry (MS) have traditionally been achieved by encoding the conformation of a protein into its mass through solution-phase chemical reactions. ${ }^{1-4}$ The location and extent of labeling is dependent on reactant concentration, reactivity, and the solvent accessibility or protection factor of the participating chemical group. While these methodologies are higher throughput than high-resolution biophysical techniques, the labeling reaction, digestion, and bioinformatic analyses required for these workflows set an upper limit on their throughput.

An emerging label-free alternative couples native MS with ion mobility spectrometry (IMS). Native MS enables condensed-phase noncovalent contacts to be retained through the ionization process and the collision cross-section (CCS) of the ion can be measured by IMS. When these ions are collisionally activated in the mass spectrometer, noncovalent bonds are dissociated, unfolding the ion, and resulting in measurable changes in CCS. The energy necessary to initiate collisioninduced unfolding (CIU) transitions is indicative of condensed-phase folding, domain structure, and ligand binding. ${ }^{5-9}$

There are several implementations of IMS analyses, and many are coupled with the means of activating ions prior to mobility separation. In fact, much of the CIU work was pioneered on drift tubes (DT) and traveling wave ion mobility spectrometry (TWIMS) instruments. ${ }^{7,10-12}$ Trapped ion mobility spectrometry (TIMS) is a more recent development and typically lacks the ability to activate ions prior to mobility separation, as in-source activation and the collision cell both occur downstream of the mobility analysis. ${ }^{13-15}$ This has recently been overcome through the addition of a second TIMS device and a pair of apertures. ${ }^{16,17}$ While promising, this is not accessible to commercial Bruker timsTOF users.

Here, we demonstrate the capability to conduct CIU on a commercially available Bruker timsTOF. Activation is accomplished through the careful manipulation of TIMS transfer DC voltages and tunnel-in pressures. This enables the systematic unfolding of native-like ions of ubiquitin, cytochrome $\mathrm{C}$, $\beta$-lactoglobulin, and carbonic anhydrase. Moreover, the inherently high resolution of the TIMS separation uncovers previously obscured unfolding intermediates.

\section{Experimental Section \\ Materials and Reagents}

Mass spectrometry grade optima water and ammonium acetate were acquired from Fisher Scientific (Waltham, MA). Ubiquitin, $\beta$-lactoglobulin, cytochrome $\mathrm{C}$, and carbonic anhydrase were all acquired from Sigma Aldrich (St. Louis, MO).

\section{Mass Spectrometry}

All proteins were dissolved to concentrations of $8 \mu \mathrm{M}$ in $100 \mathrm{mM}$ ammonium acetate and directly infused at $5 \mu \mathrm{l} / \mathrm{min}$ using a Hamilton $100 \mu \mathrm{l}$ syringe (Reno, NV) into a Bruker timsTOF (Billerica, MA). The instrument was equipped with an electrospray ionization source and the capillary voltage, endplate offset, Nebulizing gas, dry gas, and dry temperature were set to $4000 \mathrm{~V}, 400 \mathrm{~V}, 1.5 \mathrm{bar}, 4 \mathrm{l} / \mathrm{min}$, and $150{ }^{\circ} \mathrm{C}$, respectively. All CIU heatmaps were collected with a $200 \mathrm{~ms}$ mobility ramp. CIU fingerprints were collected by increasing $\Delta 6$ voltage by $5 \mathrm{~V}$ increments from 31 to $150 \mathrm{~V}$ ( $30 \mathrm{~V}$ is not allowed by the software so first step is $4 \mathrm{~V}$ ). Tunnel-in pressure was set between 2.5 and 1.5 mbar depending on the energy required to induce unfolding. Mobility accumulation was 
set to $80 \mathrm{~ms}$ for all experiments. The mobility scan ranged from $1 / \mathrm{K}_{0}=0.5-2.0 \mathrm{~V} \cdot \mathrm{s} / \mathrm{cm}^{2}$ for tunnel-in pressures of 2.0 and 1.5. At $2.5 \mathrm{mbar} 1 / \mathrm{K}_{0}=0.5-1.65 \mathrm{~V} \cdot \mathrm{s} / \mathrm{cm}^{2}$ which is required to lower $\Delta 6$ to $31 \mathrm{~V}$.

The instrument was mass and mobility calibrated with Bruker tune mix prior to all experiments and following any tunnel-in pressure changes. Mobility spectra were manually exported and then plotted with CIUsuite $2 .{ }^{18}$ To calculate collisional cross-section of measured native-like ions, mobility spectra collected with $\Delta 6=31 \mathrm{~V}$ were fitted using the multiple peak fitting tool within OriginPro 2016. All reported CCS values should be considered ${ }^{\text {TIMS }} \mathrm{CCS}_{\mathrm{N} 2}$ based on the recent recommended nomenclature. ${ }^{19}$

\section{Results and Discussion}

Trapped ion mobility utilizes the interplay of a progressively increasing electric field and a flowing gas to trap ions. The position in the $\mathrm{z}$-dimension that ions are trapped within the TIMS device is dependent on the force applied by the gaswhich is proportional to their collision cross section-and the resistive force provided by the electrical field (Figure 1A). Once oriented along this electrical field by mobility, the field is incrementally decreased eluting the most extended ions first. These eluted ions are sequentially analyzed by a downstream orthogonal time-of-flight mass analyzer.

The energy imparted to ions during the TIMS mobility analysis has been the subject of multiple studies and they identified gas pressure, accumulation time, and a series of DC potentials as critical variables to inadvertent activation. ${ }^{20-22}$ Utilizing these concepts, we recently demonstrated that through careful regulation of TIMS tunnel-in pressure and the $\triangle 6 \mathrm{DC}$ voltage setting (Figure 1A) peptide and protein ions can be controllably dissociated. ${ }^{15}$ While both our recent manuscript and these previous studies have demonstrated unfolding of protein ions, the analytical value of these processes has yet to be explored. To examine if this same process could be utilized to perform collision induced unfolding of native-like protein ions, we first examined ubiquitin at a tunnel-in pressure of 2.5 mbar.

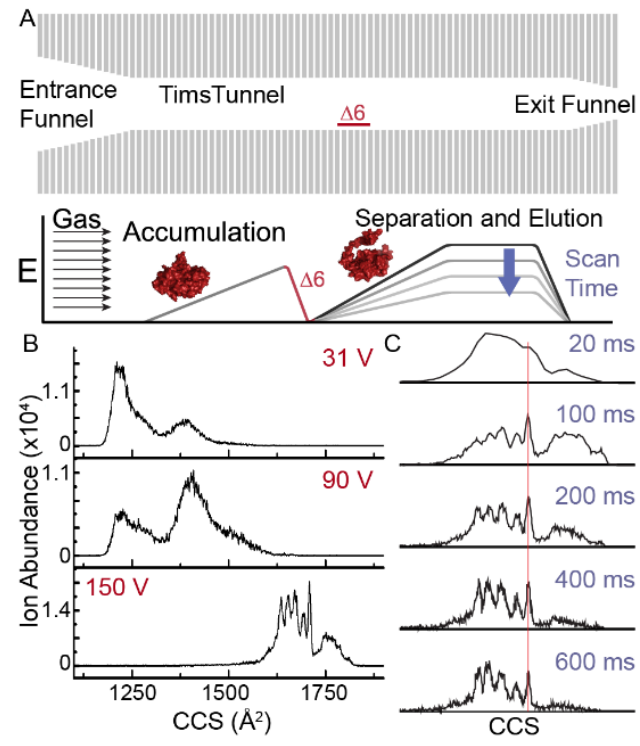

Figure 1. A) Diagram of TIMS device and plot of the applied electric field at various steps of the TIMS analysis with the location of the $\triangle 6$ DC voltage indicated on both (PDB accession codes $6 \mathrm{EYY}$ and $6 \mathrm{LKF}$ ). B) Mass resolved mobility spectra of the $6+$ charge state of ubiquitin at increasing $\Delta 6$ values. C) Mobil- ity spectra of the 6+ charge state of ubiquitin aligned at the designated point, $\Delta 6$ of $150 \mathrm{~V}$, and indicated mobility scan times.

When ubiquitin is dissolved in water and $100 \mathrm{mM}$ ammonium acetate and directly infused into the mass spectrometer, the 5+, 6+, and 7+ ions are observed (Figure $\mathbf{2 A}$ ). By lowering the TIMS transfer DC voltages $\Delta 3, \Delta 4$, and $\Delta 6$ to 70,50 , and $31 \mathrm{~V}$, respectively, we observed collision cross-sections of $1177 \AA^{2}$ for the 5+ ion and 1220,1256 , and $1389 \AA^{2}$ for the $6+$ ion of ubiquitin (Figures 1B and 2B). The 5+ and the most dominate $6+$ species are within $3 \%$ of those measured by a DT instrument in a recently published work, suggesting that native-like ions are being observed. ${ }^{23}$ When $\Delta 6$ is increased to 90 and then to $150 \mathrm{~V}$ the mobility spectrum of the $6+$ charge state of ubiquitin undergoes extensive unfolding and populates a series of unfolded states (Figure 1B).

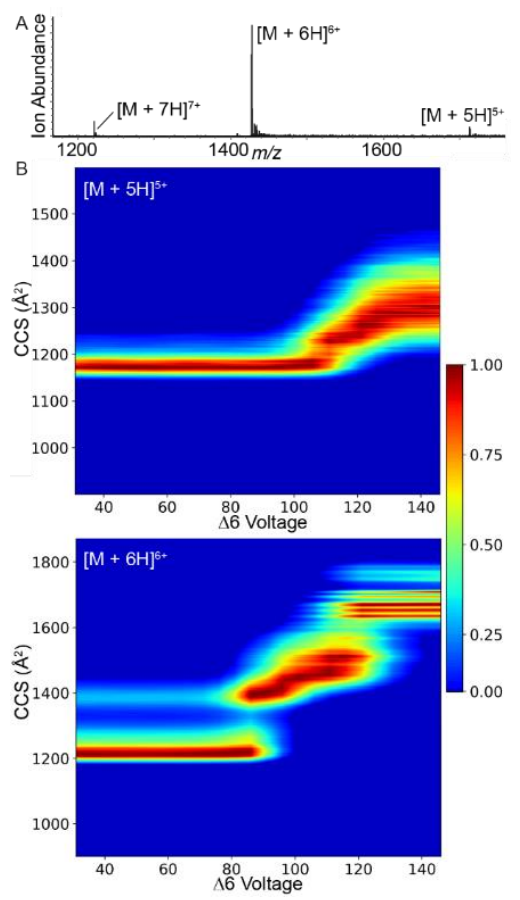

Figure 2. A) Mass spectrum of native-like ions of ubiquitin. B) CIU fingerprints of the 5+ (top) and 6+ (bottom) charge states of ubiquitin (Tunnel-in pressure $=2.5 \mathrm{mbar}$ ).

TIMS-based mobility spectrometry analyses have been shown to possess between 3 and 8 times greater resolution than those of many implementations of DT and TWIMS. ${ }^{24}$ But, unlike most other implementations of ion mobility, the resolution of the TIMS measurement can be adjusted by manipulating the mobility scan time (Figure 1A). To further explore this, ubiquitin was analyzed following accumulation with a $\Delta 6$ of $150 \mathrm{~V}$, this generated a series of closely spaced mobility features (Figure 1B). If analyzed with $20 \mathrm{~ms}$ scan time (the lowest increment of 5 available), few of the features are resolved and significant amounts of information is lost (Figure 1C). Incrementally increasing the scan time from 20 to $100,200,400$, and $600 \mathrm{~ms}$ results in increasingly resolved peaks enabling the identification of six separate unfolded species (Figure 1C). Fewer measurements can be accomplished with these longer scan times and signal to noise ratios decrease rapidly unless acquisition time is increased to compensate.

In this TIMS CIU workflow, activation of the protein ion occurs in a distinct step prior to mobility analysis, therefore 
there is a possibility of the protein ion thermalizing with the buffer gas and repopulating folded structures during extended
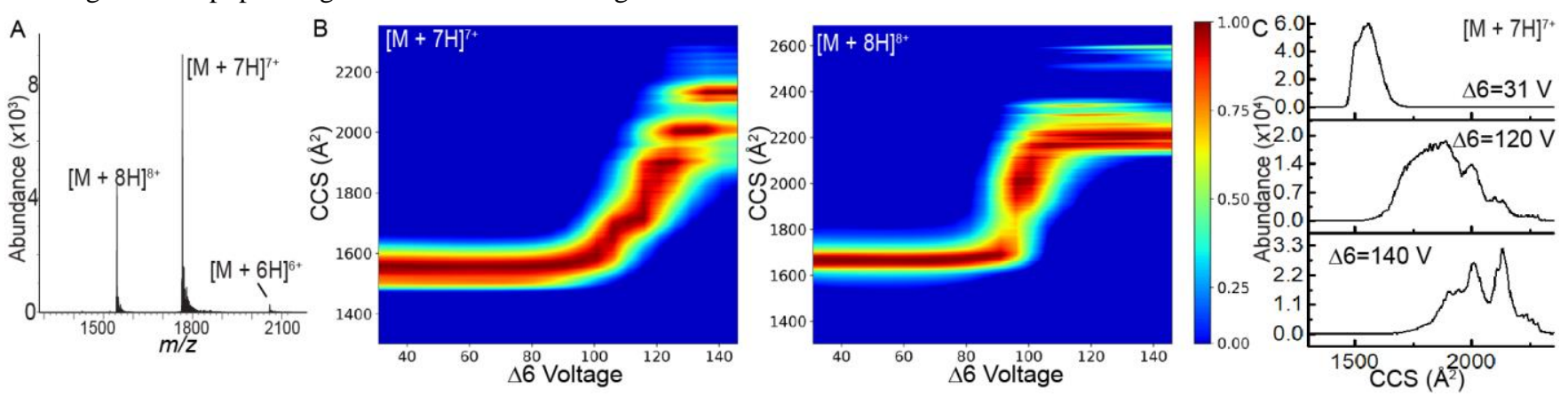

Figure 3. A) Native mass spectrum of cytochrome C. B) CIU fingerprints of the $7+$ and $8+$ charges states of cytochrome C. C) Mass resolved mobility spectra of the $7+$ charge state of cytochrome $\mathrm{C}$ following activation at the indicated $\Delta 6$. Tunnel-in pressure $=2.5$ mbar.

mobility analyses. Previous studies examining this phenomenon demonstrate refolding of activated ions within $\sim 1 \mathrm{~min}$ at pressures below $10^{-6}$ mbar and $\sim 10$ secs at $10^{-3}$ mbar. $^{25,26}$ The TIMS device operates at significantly higher pressures $(\sim 2$ mbar) than those utilized in these works and thus may accelerate this refolding further. This process may be observed in Figure 1C as the most extended feature steadily decreases in abundance and the relative distribution of the mobility features shift towards the lower CCS species as scan time is increased (Figure 1C). The absence of the most extended feature in the 20 ms scan time, however, challenges this contention and thus more work is needed to characterize this process. To balance the improved resolution of the TIMS platform and limit the possibility of refolding, a $200 \mathrm{~ms}$ scan time was chosen for all further analyses. At this scan time all species investigated in this work will be eluted after only $\sim 50 \mathrm{~ms}$ on the ramp. ${ }^{27}$

The timsTOF is not equipped with a quadrupole prior to the TIMS device, thus, all generated ions are activated simultaneously. Recent work has demonstrated that this coactivation of ions enables a multiplexed workflow that can significantly improve the thorough-put of the CIU workflow. ${ }^{28}$ Thus, when $\Delta 6$ is increased, all charge states are activated, simultaneously. Utilizing this process we increased $\Delta 6$ in $5 \mathrm{~V}$ increments, extracted the mass resolved mobility spectra of the 5 and $6+$ ions of ubiquitin, and plotted the CIU fingerprints of each charge state with CIU Suite 2 (Figure 2B). ${ }^{18}$ Both charge states demonstrate significant unfolding upon activation (Figure 2B). The 5+ charge state undergoes a single transition and resembles previously published results. ${ }^{29}$ The unfolding transitions demonstrated by the $6+$ charge state demonstrates significantly more complexity than the 5+ (Figure 2B). While this charge state has been investigated several times in literature, none are capable of resolving the complexity acquired with this technique. ${ }^{30-32}$ To find similar complexity in literature, previous data from the $7+$ charge state must be examined. This charge state displays similar complexity but only three extended structures are annotated at the highest energies utilized, while six are easily annotated with the TIMS-based technique (Figure 1C). ${ }^{16,33}$ Unfortunately, the $7+$ charge state does not have sufficient abundance to generate a CIU fingerprint.

When cytochrome $\mathrm{C}$ is ionized from a nondenaturing solution the 7 and $8+$ charges states are the most abundant species (Figure 3A). The 8+ charge state is observed as a single mobility feature at $1667 \AA^{2}$ and the $7+$ charge state has two features measuring 1502 and $1559 \AA^{2}$ (Figure 3B). The most compact feature of the $7+$ species is within $1 \%$ of values measured on a DT by Gadkari et. al. ${ }^{23}$ and the $8+$ charge state is within $1 \%$ of a recently proposed native-like structure, again suggesting the ionization conditions are sufficiently soft to generate native-like ions. ${ }^{34}$ When each charge state is subjected to increasing $\Delta 6$ voltages in $5 \mathrm{~V}$ increments, each charge undergoes extensive unfolding and samples a series of features that are unique to each charge state (Figure 3B). The unfolding pattern observed for the $7+$ ion resembles previously acquired data collected on a cyclic IM device but the improved resolution of the TIMS enables the differentiation of several conformers not identified in this previous work (Figure 3C). ${ }^{35,36}$ The $8+$ ion undergoes extensive unfolding over a narrow range of energies (Figure 3B). While this rapid conversion to a single dominate extended structure has been observed before, ${ }^{37}$ TIMS CIU uncovers two minor structural features at approximately 2300 and $2340 \AA^{2}$ that as far as we are aware, have not been previously resolved (Figure 3B).

We next applied CIU to $\beta$-lactoglobulin (BLG). When ionized from non-denaturing conditions, the 9, 8, and 7+ charge states of both the A and B isoforms are the most dominate species generated (Figure 4A). These isoforms differ by the G80D and A134V mutations. Due to the multiplexed feature of the TIMS CIU workflow when $\Delta 6$ is incrementally increased all isoforms and charge states were again activated. The 9+ charge state begins as a single feature at $2089 \AA^{2}$ which is within $5 \%$ of previously measured values. ${ }^{38}$ Unlike previous protein ions at 2.5 mbar, the ion only undergoes partial unfolding at the highest activation energy available. This resilience to unfolding is likely due to the larger mass and increased degrees of freedom available to these ions. Our recent work found that the maximum internal energy of an ion can be increased by decreasing the tunnel-in pressure and thus, we repeated the analysis of this protein at a tunnel-in pressure of 2.0 mbar. $^{15}$ At this pressure, the increase of $\Delta 6$ voltages promotes the $9+$ charge state to occupy a more extended structure (Figure 4B). The $8+$ charge state of BLG A demonstrates similar behavior at each pressure. While the multiplexed assay enabled the analysis of both BLG A and B, simultaneously, there was no significant difference measured between the two isoforms (data not shown). 

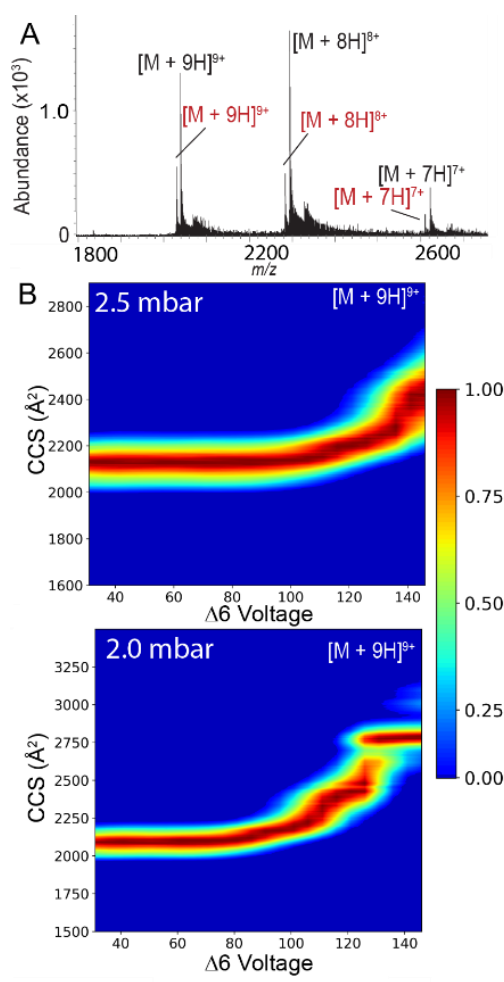

Figure 4. A) Native mass spectrum of $\beta$-lactoglobulin. The A and $\mathrm{B}$ isoforms are indicated in Black and red, respectively. B) CIU fingerprint of BLG A at a tunnel-in pressure of 2.5 (top) and 2.0 (bottom) mbar.

To further explore this mass dependence on the efficiency of the TIMS-based unfolding experiments, we next analyzed carbonic anhydrase. Native mass spectrometry of this protein results in the formation of predominately the $11+$ charge state of both the carbonic acid-bound and apo species (Figure 5 B). This charge state exists as a single mobility feature centered at $2612 \AA^{2}$ and is approximately $3 \%$ greater than a previously measured native-like ion on a TIMS instrument. ${ }^{38}$ Similarly to BLG at 2.5 mbar, the larger carbonic anhydrase at 2.0 mbar is still undergoing unfolding transitions when the maximum energy is reached (Figure 5A). To again increase the maximum energy available, the tunnel-in pressure was decreased to 1.5 mbar. Decreased tunnel-in pressures have been shown to lower the resolution of the TIMS separation but these lowered pressures are only required as the $\mathrm{m} / \mathrm{z}$ of analyte increases and resolution of the TIMS increases with $\mathrm{m} / \mathrm{z}$, limiting any detrimental effects. ${ }^{27}$ At this pressure, the $11+$ ion of carbonic anhydrase populates a series of unfolded states upon collisional activation (Figure 5A). Interestingly the dissociation of the protein:ligand complex occurs simultaneously with the first unfolding transition, suggesting that the region unfolding is important for ligand binding. Another interesting observation is the number of unfolding transitions. The number of unfolding transition is thought to be indicative of domain structure and carbonic anhydrase only possesses a single domain. ${ }^{29,39}$ This suggests that single domain protein ions may progress through a more complex series of unfolding intermediates than previously thought.
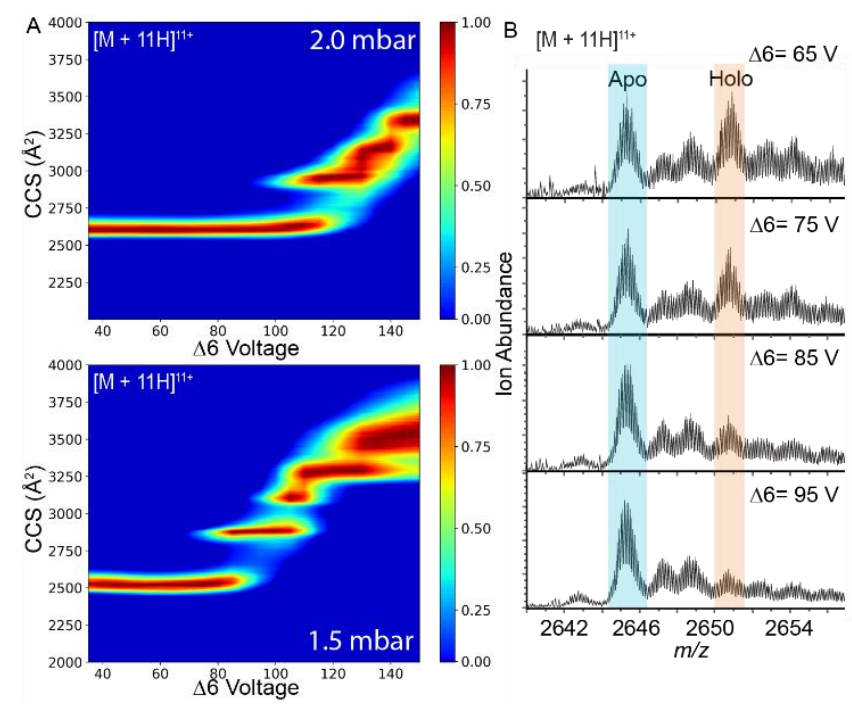

Figure 5. A) CIU fingerprints of the $11+$ charge state of Carbonic Anhydrase at tunnel-in pressure $=2.0$ mbar (top) and 1.5 mbar (bottom). B) Mass spectra of the $11+$ charge state of apoand holo-carbonic anhydrase at indicated $\Delta 6$ value.

Lastly, to examine the applicability of this TIMS CIU workflow to larger protein ions, we directly infused bovine serum albumin and while several charge states near $~ 5000$ Th were generated, no signal could be detected when the TIMS device was active (data not shown). Recently published work required the modification of the source RF generator to achieve efficient transfer of ions above $3000 \mathrm{Th} .{ }^{40}$ This appears to be a fundamental limitation of the instrument used in this manuscript. Recent work on newer generations of this instrument platform are capable of efficiently transferring higher $\mathrm{m} / \mathrm{z}$ species and future work investigating their CIU capabilities are needed. ${ }^{41}$

\section{Conclusions}

Native-like ions of ubiquitin, cytochrome $\mathrm{C}, \quad \beta$ lactoglobulin, and carbonic anhydrase were subjected to collisional activation within the TIMS device of an unmodified, commercially available Bruker timsTOF. Incremental increases in TIMS DC voltages results in the progressive unfolding of these protein ions enabling the CIU workflow on this instrumental platform. Current limitations in transfer efficiencies prohibit the analysis of proteins with native charge states greater than $3000 \mathrm{Th}$. Despite this limitation, the superior resolution of TIMS-based mobility separations could enable the detection of previously unresolved near-isochoric species. While a more systematic analysis of the interplay between tunnel-in pressure, scan time, and activation energy is undoubtably needed, these early results suggest that the TIMS platform could be a promising new tool for these increasingly utilized protein structural analyses.

\section{AUTHOR INFORMATION}

Corresponding Author

*E-mail: nborotto@unr.edu

\section{ORCID}

Nicholas B. Borotto: 0000-0003-2793-1481 


\section{Author Contributions}

The manuscript was written through contributions of all authors. All authors have given approval to the final version of the manuscript.

\section{ACKNOWLEDGMENT}

We would like to thank Varun Gadkari for his insight.

\section{REFERENCES}

(1) Konermann, L.; Pan, J.; Liu, Y.-H. Hydrogen Exchange Mass Spectrometry for Studying Protein Structure and Dynamics. Chem. Soc. Rev. 2011, 40 (3), 1224-1234. https://doi.org/10.1039/c0cs00113a.

(2) Liu, X. R.; Zhang, M. M.; Gross, M. L. Mass SpectrometryBased Protein Footprinting for Higher-Order Structure Analysis: Fundamentals and Applications. Chem. Rev. 2020, 120 (10), 4355-4454. https://doi.org/10.1021/acs.chemrev.9b00815. Limpikirati, P.; Liu, T.; Vachet, R. W. Covalent Labeling-Mass Spectrometry with Non-Specific Reagents for Studying Protein Structure and Interactions. Methods 2018, 144, 79-93. https://doi.org/10.1016/j.ymeth.2018.04.002.

(4) Engen, J. R.; Botzanowski, T.; Peterle, D.; Georgescauld, F.; Wales, T. E. Developments in Hydrogen/Deuterium Exchange Mass Spectrometry. Anal. Chem. 2021, 93 (1), 567-582. https://doi.org/10.1021/acs.analchem.0c04281.

(5) Zheng, X.; Kurulugama, R. T.; Laganowsky, A.; Russell, D. H. Collision-Induced Unfolding Studies of Proteins and Protein Complexes Using Drift Tube Ion Mobility-Mass Spectrometer. Anal. Chem. 2020, 92 (10), 7218-7225. https://doi.org/10.1021/acs.analchem.0c00772.

(6) Hong, S.; Bush, M. F. Collision-Induced Unfolding Is Sensitive to the Polarity of Proteins and Protein Complexes. J. Am. Soc. Mass Spectrom. 2019, $30 \quad$ (11), 2430-2437. https://doi.org/10.1007/s13361-019-02326-z.

(7) Hopper, J. T. S.; Oldham, N. J. Collision Induced Unfolding of Protein Ions in the Gas Phase Studied by Ion Mobility-Mass Spectrometry: The Effect of Ligand Binding on Conformational Stability. J. Am. Soc. Mass Spectrom. 2009, 20 (10), 1851-1858. https://doi.org/10.1016/j.jasms.2009.06.010.

(8) Eschweiler, J. D.; Kerr, R.; Rabuck-Gibbons, J.; Ruotolo, B. T. Sizing Up Protein-Ligand Complexes: The Rise of Structural Mass Spectrometry Approaches in the Pharmaceutical Sciences. Annu. Rev. Anal. Chem. 2017, 10 (1), 25-44. https://doi.org/10.1146/annurev-anchem-061516-045414.

(9) Dixit, S. M.; Polasky, D. A.; Ruotolo, B. T. Collision Induced Unfolding of Isolated Proteins in the Gas Phase: Past, Present, and Future. Curr. Opin. Chem. Biol. 2018, 42, 93-100. https://doi.org/10.1016/j.cbpa.2017.11.010.

(10) Freeke, J.; Bush, M. F.; Robinson, C. V.; Ruotolo, B. T. GasPhase Protein Assemblies: Unfolding Landscapes and Preserving Native-like Structures Using Noncovalent Adducts. Chem. Phys. Lett. 2012, 524, 1-9. https://doi.org/10.1016/j.cplett.2011.11.014.

(11) Pagel, K.; Hyung, S.-J.; Ruotolo, B. T.; Robinson, C. V. Alternate Dissociation Pathways Identified in Charge-Reduced Protein Complex Ions. Anal. Chem. 2010, 82 (12), 5363-5372. https://doi.org/10.1021/AC101121R.

(12) Yi Mao; Jürgen Woenckhaus; Jiri Kolafa, $\dagger$; Mark A. Ratner * and; Jarrold*, M. F. Thermal Unfolding of Unsolvated Cytochrome c: Experiment and Molecular Dynamics Simulations. J. Am. Chem. Soc. 1999, 121 (12), 2712-2721. https://doi.org/10.1021/JA980324B.

(13) Michelmann, K.; Silveira, J. A.; Ridgeway, M. E.; Park, M. A. Fundamentals of Trapped Ion Mobility Spectrometry. J. Am. Soc. Mass Spectrom. 2015, 26 (1), 14-24 https://doi.org/10.1007/s13361-014-0999-4.

(14) Ridgeway, M. E.; Lubeck, M.; Jordens, J.; Mann, M.; Park, M. A. Trapped Ion Mobility Spectrometry: A Short Review. Int. J. Mass Spectrom. 2018, 425, 22-35. https://doi.org/10.1016/j.ijms.2018.01.006.

(15) Borotto, N. B.; Graham, K. A. Fragmentation and Mobility Separation of Peptide and Protein Ions in a Trapped-Ion Mobility Device. Anal. Chem. 2021, 93 (29), 9959-9964. https://doi.org/10.1021/acs.analchem.1c01188.
Liu, F. C.; Ridgeway, M. E.; Park, M. A.; Bleiholder, C. Tandem Trapped Ion Mobility Spectrometry. Analyst 2018, 143 (10), 2249-2258. https://doi.org/10.1039/c7an02054f.

Liu, F. C.; Cropley, T. C.; Ridgeway, M. E.; Park, M. A.; Bleiholder, C. Structural Analysis of the Glycoprotein Complex Avidin by Tandem-Trapped Ion Mobility Spectrometry-Mass Spectrometry (Tandem-TIMS/MS). Anal. Chem. 2020, 92 (6), 4459-4467. https://doi.org/10.1021/acs.analchem.9b05481.

Polasky, D. A.; Dixit, S. M.; Fantin, S. M.; Ruotolo, B. T. CIUSuite 2: Next-Generation Software for the Analysis of GasPhase Protein Unfolding Data. Anal. Chem. 2019, 91 (4), 31473155. https://doi.org/10.1021/acs.analchem.8b05762.

(19) Gabelica, V.; Shvartsburg, A. A.; Afonso, C.; Barran, P.; Benesch, J. L. P.; Bleiholder, C.; Bowers, M. T.; Bilbao, A.; Bush, M. F.; Campbell, J. L.; Campuzano, I. D. G.; Causon, T.; Clowers, B. H.; Creaser, C. S.; De Pauw, E.; Far, J.; FernandezLima, F.; Fjeldsted, J. C.; Giles, K.; Groessl, M.; Hogan, C. J.; Hann, S.; Kim, H. I.; Kurulugama, R. T.; May, J. C.; McLean, J. A.; Pagel, K.; Richardson, K.; Ridgeway, M. E.; Rosu, F.; Sobott, F.; Thalassinos, K.; Valentine, S. J.; Wyttenbach, T. Recommendations for Reporting Ion Mobility Mass Spectrometry Measurements. Mass Spectrom. Rev. 2019, 38 (3), 1-30. https://doi.org/10.1002/mas.21585.

(20) Naylor, C. N.; Ridgeway, M. E.; Park, M. A.; Clowers, B. H. Evaluation of Trapped Ion Mobility Spectrometry Source Conditions Using Benzylammonium Thermometer Ions. J. Am. Soc. Mass Spectrom. 2020, 31 (7), 1593-1602. https://doi.org/10.1021/jasms.0c00151.

(21) Morsa, D.; Hanozin, E.; Eppe, G.; Quinton, L.; Gabelica, V.; Pauw, E. De. Effective Temperature and Structural Rearrangement in Trapped Ion Mobility Spectrometry. Anal. Chem. 2020, 92 (6), 4573-4582. https://doi.org/10.1021/acs.analchem.9b05850.

(22) Bleiholder, C.; Liu, F. C.; Chai, M. Comment on Effective Temperature and Structural Rearrangement in Trapped Ion Mobility Spectrometry. Anal. Chem. 2020, 92 (24), 1632916333. https://doi.org/10.1021/acs.analchem.0c02052.

(23) Gadkari, V. V.; Ramírez, C. R.; Vallejo, D. D.; Kurulugama, R. T.; Fjeldsted, J. C.; Ruotolo, B. T. Enhanced Collision Induced Unfolding and Electron Capture Dissociation of Native-like Protein Ions. Anal. Chem. 2020, 92 (23), 15489-15496. https://doi.org/10.1021/acs.analchem.0c03372.

(24) Silveira, J. A.; Ridgeway, M. E.; Park, M. A. High Resolution Trapped Ion Mobility Spectrometery of Peptides. Anal. Chem. 2014, 86 (12), 5624-5627. https://doi.org/10.1021/ac501261h.

(25) Horn, D. M.; Breuker, K.; Frank, A. J.; McLafferty, F. W. Kinetic Intermediates in the Folding of Gaseous Protein Ions Characterized by Electron Capture Dissociation Mass Spectrometry. J. Am. Chem. Soc. 2001, 123 (40), 9792-9799. https://doi.org/10.1021/ja003143u.

(26) Badman, E. R.; Myung, S.; Clemmer, D. E. Evidence for Unfolding and Refolding of Gas-Phase Cytochrome c Ions in a Paul Trap. J. Am. Soc. Mass Spectrom. 2005, 16 (9), 1493-1497. https://doi.org/10.1016/j.jasms.2005.04.013.

(27) Hernandez, D. R.; DeBord, J. D.; Ridgeway, M. E.; Kaplan, D. A.; Park, M. A.; Fernandez-Lima, F. Ion Dynamics in a Trapped Ion Mobility Spectrometer. Analyst 2014, 139 (8), 1913-1921. https://doi.org/10.1039/c3an02174b.

(28) Vallejo, D. D.; Polasky, D. A.; Kurulugama, R. T.; Eschweiler, J. D.; Fjeldsted, J. C.; Ruotolo, B. T. A Modified Drift Tube Ion Mobility-Mass Spectrometer for Charge-Multiplexed CollisionInduced Unfolding. Anal. Chem. 2019, 91 (13), 8137-8146. https://doi.org/10.1021/acs.analchem.9b00427.

(29) Zhong, Y.; Han, L.; Ruotolo, B. T. Collisional and Coulombic Unfolding of Gas-Phase Proteins: High Correlation to Their Domain Structures in Solution. Angew. Chemie - Int. Ed. 2014, 53 (35), 9209-9212. https://doi.org/10.1002/anie.201403784.

(30) Wagner, N. D.; Kim, D.; Russell, D. H. Increasing Ubiquitin Ion Resistance to Unfolding in the Gas Phase Using Chloride Adduction: Preserving More "Native-Like" Conformations Despite Collisional Activation. Anal. Chem. 2016, 88 (11), 5934-5940. https://doi.org/10.1021/acs.analchem.6b00871.

(31) Kit, M. C. S.; Shepherd, S. O.; Prell, J. S.; Webb, I. K. Experimental Determination of Activation Energies for Covalent Bond Formation via Ion/Ion Reactions and Competing Processes. J. Am. Soc. Mass Spectrom. 2021, 21, 45. 

Reactivity of Gaseous Ubiquitin Ions Electrosprayed from Aqueous and Denaturing Solutions. J. Am. Soc. Mass Spectrom.
2020,
31
(5),
1037-1046.

. Solution Dependence of the Collisional Activation of Ubiquitin $[\mathrm{M}+7 \mathrm{H}](7+)$ Ions. J. Am. Soc. Mass Spectrom. 2014, 21-26. https://doi.org/10.1007/s13361-014-0834-y.

(34) Molano-Arevalo, J. C.; Fouque, K. J. D.; Pham, K.; Miksovska, J.; Ridgeway, M. E.; Park, M. A.; Fernandez-Lima, F. Characterization of Intramolecular Interactions of Cytochrome c Using Hydrogen Deuterium Exchange - Trapped Ion Mobility Spectrometry- Mass Spectrometry and Molecular Dynamics. Anal. Chem. 2017, $89 \quad$ (17), 8757 https://doi.org/10.1021/ACS.ANALCHEM.7B00844.

(35) Eldrid, C.; Ujma, J.; Kalfas, S.; Tomczyk, N.; Giles, K.; Morris, M.; Thalassinos, K. Gas Phase Stability of Protein Ions in a Cyclic Ion Mobility Spectrometry Traveling Wave Device. Anal. Chem. 2019, $91 \quad$ (12), 7554-7561. https://doi.org/10.1021/ACS.ANALCHEM.8B05641.

(36) Eldrid, C.; Ben-Younis, A.; Ujma, J.; Britt, H.; Cragnolini, T.; Kalfas, S.; Cooper-Shepherd, D.; Tomczyk, N.; Giles, K.; Morris, M.; Akter, R.; Raleigh, D.; Thalassinos, K. Cyclic Ion Mobility-Collision Activation Experiments Elucidate Protein Behavior in the Gas Phase. J. Am. Soc. Mass Spectrom. 2021, 32 (6), 1545-1552. https://doi.org/10.1021/jasms.1c00018.

(37) Yin, V.; Konermann, L. Probing the Effects of Heterogeneous Oxidative Modifications on the Stability of Cytochrome c in Solution and in the Gas Phase. J. Am. Soc. Mass Spectrom. 2020, 32 (1), 73-83. https://doi.org/10.1021/JASMS.0C00089.

(38) Benigni, P.; Marin, R.; Molano-Arevalo, J. C.; Garabedian, A.; Wolff, J. J.; Ridgeway, M. E.; Park, M. A.; Fernandez-Lima, F. Towards the Analysis of High Molecular Weight Proteins and Protein Complexes Using TIMS-MS. Int. J. Ion Mobil. Spectrom. $2016 \quad 192 \quad \mathbf{2 0 1 6}, 19 \quad 1$ (2), 95-104. https://doi.org/10.1007/S12127-016-0201-8.

(39) Eschweiler, J. D.; Martini, R. M.; Ruotolo, B. T. Chemical Probes and Engineered Constructs Reveal a Detailed Unfolding Mechanism for a Solvent-Free Multidomain Protein. J. Am. Chem. Soc. 2017, 139 (1), 534-540. https://doi.org/10.1021/jacs.6b11678.

(40) Panczyk, E. M.; Snyder, D. T.; Ridgeway, M. E.; Somogyi, Á.; Park, M. A.; Wysocki, V. H. Surface-Induced Dissociation of Protein Complexes Selected by Trapped Ion Mobility Spectrometry. Anal. Chem. 2021, 93 (13), 5513-5520. https://doi.org/10.1021/ACS.ANALCHEM.0C05373.

(41) Larson, E. J.; Roberts, D. S.; Melby, J. A.; Buck, K. M.; Zhu, Y.; Zhou, S.; Han, L.; Zhang, Q.; Ge, Y. High-Throughput Multi-Attribute Analysis of Antibody-Drug Conjugates Enabled by Trapped Ion Mobility Spectrometry and Top-Down Mass Spectrometry. Anal. Chem. 2021, 93 (29), 10013-10021. https://doi.org/10.1021/acs.analchem.1c00150.

Insert Table of Contents artwork here

\section{TIMS}

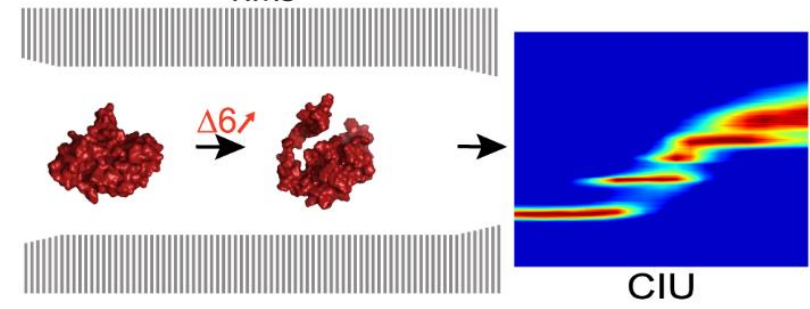

